\title{
5 Bewusstseinserweiterung beim Gebrauch psychoaktiver Substanzen
}

\author{
Rolf Verres
}

Wir nehmen die äußere und die innere Welt mit unseren Sinnen wahr, wir haben Gefühle, wir denken nach, schmieden Pläne und bewerten unser Verhalten und das Verhalten anderer. Das Gedächtnis ermöglicht uns, frühere Ereignisse mit der Gegenwart in Beziehung zu setzen, aus unseren Erfahrungen zu lernen, flexibel und kreativ zu werden und eine mehr oder weniger gelingende Lebenskunst im Gemeinwesen zu entwickeln, die auch Vorstellungen über die Zukunft einbezieht. Dies alles bedeutet eine permanente seelische und kulturelle Weiterentwicklung.

Könnten die Menschen mit diesen Fähigkeiten ihres Bewusstseins nicht einfach zufrieden sein?

Das Streben nach Bewusstseinserweiterung ist manchmal mit Risiken und Nebenwirkungen verbunden. Man weiß selten, wohin „,andere“ Arten des eigenen Bewusstseins, die man bisher noch nicht kannte, einen führen werden. Das gilt ganz besonders für den Konsum psychoaktiver Substanzen.

Allerdings: Viele Menschen spüren im tiefen Inneren eine Ahnung davon, dass es neben der Selbstverständlichkeit der Alltagsroutinen noch andere Erfahrungsmöglichkeiten geben könnte. Das erlebt man, ob man es will oder nicht, bei den vielen Varianten des Träumens. Es gibt auch die Märchen, die Romane, Opern und Filme aus anderen Welten, die Tagträume und die Fantasien. Darin erleben wir relativ risikofreie Möglichkeiten, uns über die Alltagsrealität hinwegzusetzen .

Wie Andrew Weil in seinem Buch „The Natural Mind“ (1972, deutsche Ausgabe „Drogen und höheres Bewusstsein“, 2000) kritisierte, wissen viele Menschen, die von selbst erlebter Bewusstseinserweiterung sprechen, nicht so recht, was sie eigentlich damit meinen. Beispielsweise sind Gefühle von körperlicher Leichtigkeit und Zeit- 
losigkeit, die von Drogen-Usern beschrieben werden, oft besonders angenehm; sie lassen sich aber durch viele weitere und heterogene Techniken auslösen und gehören überdies zu den Empfindungen, die fast jeder auch schon in der Kindheit kannte, sodass man etwas nüchtern sagen könnte:

Als Erweiterung bezeichnen wir alles in unserem Seelenleben, was wir bisher noch nicht kannten oder wozu wir durch Vergessen oder Verdrängen den Zugang verloren hatten und ihn nun durch eine außergewöhnliche Erfahrung wiedergefunden haben.

Die meisten psychotherapeutischen Konzepte sind dem Anliegen gewidmet, noch nicht entdeckte oder brachliegende Ressourcen neu zu entdecken, neu zu beleben und vor allem neu für die Lebenskunst zu nutzen.

Dazu sind Visionen bedeutsam. Sie können spontan auftreten, wie z.B. als drogeninduzierte Halluzination, sie können aber auch gezielt mit mentalen Methoden angestrebt werden, wie z.B. im vision quest mit gleichzeitigem Reizentzug. Viele in unserem Seelenleben eingeprägte Bilder hindern uns daran, zu dem zu werden, der wir sein könnten. Dazu gehören Blickverengungen, stereotype Menschen-, Welt- und Feindbilder oder auch die Gewohnheit, die Realitäten so hinzunehmen, wie sie sind, statt Veränderungen in Betracht zu ziehen.

Wenn sich nun beim Träumen, in Tagträumen, bei drogeninduzierten Halluzinationen oder bei weiteren „Reisen in andere Wirklichkeiten“ ein gewohnter Blick zu weiten beginnt, ist das schon Bewusstseinserweiterung?

Zumindest sind es oft glückliche Momente, in denen einem „das Herz aufgeht“, Ideen zu sprudeln beginnen oder eine nie gekannte Faszination erreicht wird.

Es gibt auch die anstrengenden Wege zur Bewusstseinserweiterung. Die von Freud begründete Psychoanalyse wird als eine Methode der Bewusstseinserweiterung angesehen, da sie Möglichkeiten der Bewusstmachung unbewusster Anteile des Seelenlebens nutzt. Dazu gehört auch das mühsame Arbeiten am sogenannten Widerstand. Allein schon die Fähigkeit, Träume mit sinnvollem Bezug zum eigenen Leben zu deuten, erfordert ein gewisses Maß an Know-how und an Arbeit. Der Träumer fantasiert Lebensthemen wie in einem Theater, wobei er gleichzeitig Autor, Regisseur, Akteur und Zuschauer ist. Im Alltag verdrängte Lebensthemen werden im Traum wieder eingeblendet. Die zu (hoffentlich) neuen Erkenntnissen führende analytische Arbeit erfordert ein sich Einlassen auf ziemlich komplexe Zusammenhänge.

Sowohl Trauminhalte als auch Halluzinationen (oder Visionen) unter der Einwirkung psychoaktiver Substanzen können als etwas angesehen werden, das den betreffenden Menschen überflutet, ihm also als etwas widerfährt, auf das er keinen bewussten Einfluss zu haben scheint. Man sagt: „Es träumte mir“. Die Traumdeutung wird erst dann zu einer Bewusstseinserweiterung beitragen, wenn sie sowohl mit ihren faszinierenden wie auch verunsichernden Aspekten ergebnisoffen und gleichzeitig selbstkritisch in einer Weise praktiziert wird, bis sie als Fortschritt der Selbsterkenntnis gewertet werden kann. Gut, dass es geschulte Psychotherapeuten gibt, die hierbei wie eine Hebamme auf mentalem Gebiet professionelle Hilfestellung bieten können.

Eine Sonderform des Träumens ist das sogenannte luzide Träumen, bei dem der Mensch weiß, dass er träumt und während des Träumens aktiv Einfluss auf die Traumge- 
schichte nehmen kann. Voraussetzung hierfür ist, dass das sogenannte beobachtende Ich während des Träumens zumindest teilweise erhalten bleibt. Diese Fähigkeit hat auch für die Integration drogeninduzierter veränderter Bewusstseinszustände eine sehr wichtige Bedeutung. Sie hängt von Art und Dosis der psychoaktiven Substanz ab. Je nach Substanz und Dosis können die Möglichkeiten eines beobachtenden Ichs erhalten bleiben oder verlorengehen. Konzepte von Stephen LaBerge (1987) und Brigitte Holzinger (2007), ganz besonders aber auch die Konzepte zum katathymen Bilderleben von Hanscarl Leuner (1985) laufen allesamt darauf hinaus, während des veränderten Bewusstseinszustandes die beobachtenden und zur Steuerung fähigen Ich-Anteile bewusst zu machen und darin zu trainieren, eine angestrebte Bewusstseinserweiterung durch mentale Erarbeitung kontrollierender Bewusstseinstechniken anzustreben.

Eine besondere Konstellation ergibt sich bei Albträumen und drogeninduzierten Horrortrips, die anstelle einer angestrebten Bewusstseinserweiterung eintreten können.

Albträume und Horrortrips sind typische Beispiele für veränderte Bewusstseinszustände, bei denen die Steuerungsfähigkeit des beobachtenden Ichs überfordert ist und versagt.

Hiermit angemessen umzugehen erfordert von professioneller Seite eine außerordentlich differenzierte Kompetenz, die z.B. durch Selbsterfahrung mit psychoaktiven Substanzen mit Intervision und/oder Supervision gefördert werden kann. Im Buch unserer Heidelberger Arbeitsgruppe über „Therapie mit psychoaktiven Substanzen“ (Jungaberle et al. 2008) werden viele dieser Techniken ausführlich geschildert.

$\mathrm{Zu}$ den besonders vielversprechenden Techniken gehört das Prinzip des Erweckens aus veränderten Bewusstseinszuständen im richtigen Augenblick.

\begin{abstract}
Der niederländische Psychiater Jan Bastiaans, Professor für psychosomatische Medizin an der staatlichen Universität in Leiden, veranschaulichte dieses Prinzip am Beispiel der Psychotherapie ehemaliger Insassen von Nazi-Konzentrationslagern, die er unter LSD-Einfluss mit Begleitung des Psychotherapeuten die posttraumatischen grauenhaften Albträume noch einmal durchleben ließ und unter der Wirkung von LSD die nachträglich aufgebauten aber nicht hilfreichen Abwehrmechanismen aufzuweichen versuchte. Voraussetzung hierfür war, dass die gewählte Dosis von LSD einerseits ein tiefes Eintauchen in die traumatischen Erinnerungen förderte, aber gleichzeitig die Fähigkeit des Patienten, während des LSD-unterstützten Traumerlebens mit dem Therapeuten sprachlich in Kontakt zu bleiben, trainiert wurde (Bastiaans 1987, 2000).
\end{abstract}

Von den Menschen, die mithilfe psychoaktiver Substanzen eine Bewusstseinserweiterung erleben wollen, wissen wahrscheinlich nur wenige Bescheid über die Möglichkeiten des Geistes, die dem „Trip“ eine Richtung geben können. Viele Menschen führen Bewusstseinserweiterung dann einzig auf die konsumierte Drogen zurück und nicht auf die eigene Person, die mit der Gestaltung von Set und Setting das, was geschieht, ganz wesentlich beeinflussen kann. Hier sehe ich eine wichtige Aufgabe von Psychotherapeuten, denjenigen Menschen, die Bewusstseinserweiterung suchen, nicht erst in den psychotherapeutischen Kontexten von Kliniken, sondern bereits im Sinne von harm reduction beim Gebrauch psychoaktiver Substanzen ganz konkrete Anleitungen darüber zu geben, was hierbei zu beachten ist. 
In seinem Buch „The unfolding self - varieties of transformative experience“ behauptet der deutsch-amerikanische Bewusstseinsforscher Ralph Metzner (1998), man könne das, was wir als die Realität empfinden, ebenso als einen Traum oder eine Trance bezeichnen. Plausibel wird dieser Gedanke dann, wenn wir das selbstverständliche Funktionieren am Fließband oder im Haushalt, auch das unkritische Hinnehmen von Alltagsrealitäten und Gewohnheiten, denen wir uns automatisch überlassen und bei denen wir uns wie im Hamster-Laufrad fühlen, versuchsweise als Trance betrachten.

Ist das Hineingehen in eine intendierte Trance bewusstseinserweiternd oder ist das Aussteigen aus einer gewohnheitsmäßigen Alltags-Trance bewusstseinserweiternd?

Ralph Metzner meint hierzu, Bewusstseinserweiterung sei ein Erwachen. Die Metapher des Erwachens findet sich im Hinduismus, Buddhismus, Sufismus, auch im Christentum, beispielsweise im Pfingstfest zu Ehren des Heiligen Geistes. Metzner versteht unter Erwachen ein Aussteigen aus dem „Schleier von Illusionen“, in dem sich viele Menschen eingenistet haben. Visionen könnten zu einer „Reinigung der Pforten der Wahrnehmung “ führen, wie es in mystischen und psychedelischen $\mathrm{Zu}$ ständen manchmal empfunden wird, jedenfalls dann, wenn „Klarsehen“ als ein transformierendes Ereignis empfunden wird.

Ein Hauptthema der Literatur über Bewusstseinserweiterung ist immer wieder der Wunsch, unverbundene, fragmentierte Anteile der Psyche in ein integriertes Ganzes transformieren zu wollen.

Bewusstseinserweiterung ist nicht in erster Linie ein Abheben in ein neues Universum, sondern notwendig ist zugleich das Halten eines Kontaktes mit dem Boden, mit dem Alltagsbewusstsein.

Daher werden in der psycholytischen und psychedelischen Therapie bevorzugt Substanzen wie MDMA und LSD verwendet, bei denen erfahrungsgemäß (je nach Dosis) ein beobachtender Ich-Anteil erhalten bleibt oder zumindest in Ritualen, die den „Trip“ unterbrechen sollen, reaktiviert wird (Jungaberle et al. 2008).

Die Vorstellung von Erleuchtung taucht immer wieder in Diskursen zur Bewusstseinserweiterung auf. Nach meiner Meinung macht es durchaus Sinn, entsprechenden Vorstellungen zunächst einmal wohlwollend zu begegnen. Konnotationen und Metaphern über Feuer, Leuchten, Inspiration usw. haben etwas mit Wärme zu tun, manchmal auch mit Glückseligkeit.

Es gibt aber auch „Erleuchtungsfallen“ (Klaus Horn in Reiter und Bucher 2008, Scharfetter 1991 und Verres in Vorbereitung). Religiös-spirituelle Krisen können durch Drogenkonsum ausgelöst werden und verhängnisvolle Bedeutungen erlangen, wie z.B. bei angstvoller Ich-Auflösung oder narzisstischem Größenwahn und überstarker Identifizierung mit dem Göttlichen. Die Person kann den Bodenkontakt verlieren und eine Furcht entwickeln, verrückt geworden zu sein in einer Lebenswelt, die an nüchterner Rationalität orientiert ist. In seinem Buch „Der spirituelle Weg und seine Gefahren“ berichtete der Zürcher Psychiater Christian Scharfetter (1991) über seine Erfahrungen mit Suchern, Erschöpften, Zweiflern, Verzagten und Geängstigten, die nach dem Tod früherer Bezugssysteme eine Art Neugeburt suchen, aber dennoch keine Befreiung oder Erlösung finden. Auch wenn das normale Tageswachbewusstsein in einer spirituellen Wertehierarchie relativiert wird, muss es weiterhin bedeutsam bleiben. 
Ebenso wie bei psychoaktiven Substanzen scheint es auch bei spirituellen Erlebnissen so etwas wie eine „Überdosis“ zu geben, die zu einer beängstigenden Desintegration der für die Alltagsbewältigung notwendigen Ich-Funktionen führen kann.

„Das Wünschenswerte ist nicht ein Austritt aus dem Tageswachbewusstsein in das Überbewusstsein, sondern die allmähliche Ausdehnung des Bewusstseins mit der Integration immer weiterer Bewusstseinsbereiche“ (Scharfetter 1991, S. 32).

Im Zen-Buddhismus sagt man über jemanden, der sich etwas auf eigene Erleuchtungserfahrungen einbildet und viel Aufhebens davon macht: „Er stinkt nach Erleuchtung“. Erst wenn sich dieser „Gestank“ gelegt hat, erkennt man dem Übenden im Zen wahre Erleuchtung zu (Rhyner 1991).

Es sei nun noch die Frage gestellt, wie man eigentlich zu Wertungen veränderter Bewusstseinszustände kommen kann.

- Kann eine Bewusstseinsverengung im Sinne von Fokussierung der Aufmerksamkeit nicht genauso wichtig für die seelische Weiterentwicklung sein wie all das, was man unter Bewusstseinserweiterung zu verstehen versucht?

- Ist es nicht vielleicht sogar anmaßend, wenn ein Mensch, der einen bisher unbekannten Bewusstseinszustand erreicht hat, von Bewusstseinserweiterung spricht und damit zugleich diejenigen abwertet, die im banalen Alltagsbewusstsein hängen geblieben sind wie ein sich im Schlamm abstrampelnder Frosch?

Hierzu ist die Beobachtung interessant, dass Menschen, die nach Erfahrungen mit psychoaktiven Substanzen eine spirituelle Richtung einschlagen, sich früher oder später nach anderen Methoden umsehen, um ihre Erfahrungen aufrecht zu erhalten. Andrew Weil (200o) sagt hierzu:

„Es gibt viele, die lange Zeit Drogen genommen haben und diese aufgeben, um zu meditieren. Es gibt aber niemanden, der lange Zeit meditiert hat und die Meditation aufgeben würde, um ein acid head, ein gewohntheitsmäßiger LSD-Konsument, zu werden. Diese Beobachtung unterstützt das Argument, dass die Highs, die durch Meditation erlangt werden können, besser sind als die Highs, die durch Drogen erlangt werden können - ein Argument, das keinen moralischen, sondern einen rein praktischen Sinn hat."

Gerade wenn Drogen wünschenswerte Bewusstseinszustände auslösen, können sie die Illusion bestärken, dass diese Zustände nicht aus der inneren, sondern aus der äußeren Wirklichkeit entstehen.

Es gibt unendlich viele verschiedene Vorstellungen und Wege zur Bewusstseinserweiterung. Veränderte Bewusstseinszustände sind nicht automatisch mit Bewusstseinserweiterung gleichzusetzen, auch wenn viele Konsumenten psychoaktiver Substanzen das so ausdrücken. Eine momentane Bewusstseinsveränderung, z.B. als HighGefühl, das man zuvor noch nicht kannte, macht noch keine nachhaltige Bewusstseinserweiterung aus. Selbst die Definition von Bewusstseinserweiterung von Carl Gustav Jung im Jahre 1928 ist eher von Subjektivität gekennzeichnet als von konsensfähiger Objektivität. Er bezeichnete mit dem Begriff

„eine Bewusstwerdung, welche gar nicht anders als durch Unterscheidung zu Stande kommen kann. Ein Fortschritt beginnt daher immer mit Individuation, d.h., dass ein einzelner seiner Vereinzelung bewusst, einen neuen Weg durch bisher Unbetretenesbahnt. [...] Insofern es ihm gelingt, seine erweiterte Bewusstheit zu kollektiver Geltung zu bringen, gibt es durch Gegensatzspannungjene Anregung, welcher die Kultur zu ihrem weiteren Fortschritt bedarf". 
Dies ist ein hoher Anspruch, wobei die Beziehung zwischen Individuum und Kultur in den Mittelpunkt gestellt wird.

Viele Wege zur Bewusstseinserweiterung sind mit Abenteuerlust verbunden. Zu jeglicher Abenteuerreise gehören eine gute Vorbereitung und Ausrüstung, sei es bei Wildwasserfahrten, bei Reisen in die Tropen oder beim Bergsteigen. Auch hier muss man sich die Gefährten gut aussuchen, Landkarten oder ein Navigationssystem besorgen, gute Schuhe, einen Schlafsack und vielleicht auch ein Zelt mitnehmen, beim Essen und Trinken auf das Risiko von Infektionen achten und sich über das Wetter und gefährliche Tiere wie Moskitos, Bären oder Schlangen informieren.

\section{Literatur}

Bastiaans I (1987) Klinisch-therapeutische Erfahrungen mit psychoaktiven Substanzen. In: Schlichting M, Leuner $\mathrm{H}$ (Hrsg.) 2. Symposion über psychoaktive Substanzen und veränderte Bewusstseinszustände in Forschung und Therapie. Verlag Wissenschaft und Bildung. Berlin

Bastiaans I (2000) The use of hallucinogenic Drugs in Psychosomatic Therapy. In: Schlichting, M. (Hg.). Welten des Bewusstseins. Pränatale Psychologie und psycholytische Therapie. Band 10. Verlag Wissenschaft und Bildung. Berlin

Holzinger B (2007) Anleitung zum Träumen. Klett-Cotta. Stuttgart

Jung CG (1928) Gesammelte Werke Band 7, S. 149, Orig. 1928

Jungaberle H, Gasser P, Weinhold I und Verres R (Hrsg.) (2008) Therapie mit psychoaktiven Substanzen - Praxis und Kritik der Psychotherapie mit LSD, Psilocybin und MDMA. Verlag Hans Huber. Bern

LaBerge S (1987) Hellwach im Traum, höchste Bewusstheit im tiefen Schlaf. Jungfermann. Paderborn.

Leuner H-C (1985) Lehrbuch des katathymen Bilderlebens. Verlag Hans Huber. Stuttgart Bern.

Metzner R (1998) The unfolding self - varities of transformative experience. Origin Press 1998. Novato/CA.

Reiter A, Bucher A (Hrsg.) (2008) Psychologie - Spiritualität - Interdisziplinär (173-176). Verlag Dietmar Klotz. Eschborn

Rhyner (1991) Zen Krankheit. In: Scharfetter C (1991) Der spirituelle Weg und seine Gefahren, Ferdinand Enke Verlag. Stuttgart, S. 75

Scharfetter C (1991) Der spirituelle Weg und seine Gefahren, Ferdinand Enke Verlag. Stuttgart

Verres $\mathrm{R}$ (in Vorbereitung) Abenteuer in den Welten des Bewusstseins - Erfahrungen aus 12 Jahren Drogenforschung

Weil A (2000) Drogen und höheres Bewusstsein (Original 1992) AT Verlag. Aarau, Schweiz

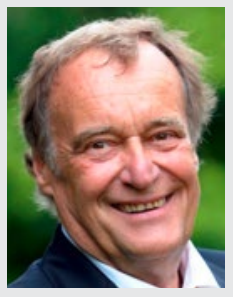

\section{Prof. Dr. med. Dipl.-Psych. Rolf Verres}

Facharzt für psychotherapeutische Medizin, Ordinarius und Ärztlicher Direktor des Instituts für Medizinische Psychologie an der Universitätsklinik Heidelberg bis zur Emeritierung im Jahr 2013.

Mitbegründer und Vorstandsmitglied des Europäischen Collegiums für Bewusstseinsstudien ECBS.

Forschungsschwerpunkte: Subjektive Theorien, Psychoonkologie, Musiktherapie, Gebrauch und Missbrauch psychoaktiver Substanzen. 